\begin{tabular}{|c|l|}
\hline Title & Structure of the pairing gap from orbital nematic fluctuations \\
\hline Author(s) & A gatsuma, Tomoaki; Y amase, Hiroy uki \\
\hline Citation & $\begin{array}{l}\text { Physical Review B, 94(21), 214505 } \\
\text { https://doi.org/10.1103/PhysRevB.94.214505 }\end{array}$ \\
\hline Issue Date & 2016-12-09 \\
\hline Doc URL & http://hdl.handle.net/2115/64379 \\
\hline Rights & 2016 A merican Physical Society \\
\hline Type & article \\
\hline File Information & PhysRevB.94.214505.pdf \\
\hline
\end{tabular}

Instructions for use 


\title{
Structure of the pairing gap from orbital nematic fluctuations
}

\author{
Tomoaki Agatsuma and Hiroyuki Yamase \\ Department of Condensed Matter Physics, Graduate School of Science, Hokkaido University, Sapporo 060-0810, Japan \\ and National Institute for Materials Science, Tsukuba 305-0047, Japan \\ (Received 9 September 2016; revised manuscript received 7 November 2016; published 9 December 2016)
}

\begin{abstract}
We study superconducting instability from orbital nematic fluctuations in a minimal model consisting of the $d_{x z}$ and $d_{y z}$ orbitals, and choose model parameters which capture the typical Fermi surface geometry observed in iron-based superconductors. We solve the Eliashberg equations down to low temperatures with keeping the renormalization function and a full momentum dependence of the pairing gap. When superconductivity occurs in the tetragonal phase, we find that the pairing gap exhibits a weak momentum dependence over the Fermi surfaces. The superconducting instability occurs also inside the nematic phase. When the $d_{x z}$ orbital is occupied more than the $d_{y z}$ orbital in the nematic phase, a larger (smaller) gap is realized on the Fermi-surface parts where the $d_{x z}\left(d_{y z}\right)$ orbital component is dominant, leading to a substantial momentum dependence of the pairing gap on the hole Fermi surfaces. On the other hand, the momentum dependence of the gap is weak on the electron Fermi surfaces. We also find that while the leading instability is the so-called $s_{++}$-wave symmetry, the second leading one is $d_{x^{2}-y^{2}}$-wave symmetry. In particular, these two states are nearly degenerate in the tetragonal phase, whereas such quasidegeneracy is lifted in the nematic phase and the $d_{x^{2}-y^{2}}$-wave symmetry changes to highly anisotropic $s$-wave symmetry.
\end{abstract}

DOI: 10.1103/PhysRevB.94.214505

\section{INTRODUCTION}

The mechanism of high- $T_{c}$ superconductivity is one of major interests in condensed matter physics. In particular, iron-based superconductors (FeSCs) attract great interest [1]. The typical phase diagram of FeSCs [2] contains four phases: normal metallic phase, superconductivity (SC), spin-densitywave (SDW), and nematic phase [3]. Because of the proximity to the SDW phase, it is widely discussed that SC can be mediated by spin fluctuations [4-6]. On the other hand, FeSCs are characterized by multibands and thus SC mediated by orbital fluctuations is also discussed as another mechanism of SC $[7,8]$.

How about a role of the nematic phase for SC? Since SC occurs closer to the nematic than the SDW phase, it is easily expected that nematic fluctuations also play an important role to drive SC. While the nematic instability is accompanied by a structural phase transition from a tetragonal to an orthorhombic phase, the nematic phase is believed to be driven by electronic degrees of freedom, not by lattice degrees. Considering that the nematic phase is associated with breaking of the orientational symmetry and keeping the translational symmetry unbroken, strong nematic fluctuations are expected to occur around zero momentum near the nematic transition. In fact, such strong nematic fluctuations were directly observed by electronic Raman spectroscopy [9]. A possible SC from nematic fluctuations is therefore distinguished from the spin [4-6] and orbital $[7,8]$ fluctuation mechanisms because the latter two mechanisms are concerned with fluctuations of a large momentum transfer characterized typically by Fermi surface (FS) nesting.

The origin of the nematic phase is under debate [10]. There are three possible nematic orders: charge [11-13], spin [14], and orbital $[15,16]$ nematicity. The latter two possibilities, namely spin [17,18] and orbital [19-22] nematic order, are mainly discussed. Since there is a linear coupling between spin and orbital nematic orders, one order necessarily leads to the other [10]. It is therefore not easy to distinguish between these two orders in experiments. Theoretically it turned out that the spin nematic phase is subject to a severely restricted property near the SDW phase [23], which may serve to identify the origin of the nematic order.

We focus on the orbital nematic scenario in this paper. Orbital nematic fluctuations lead to the so-called $s_{++}$-wave symmetry in the sense that it is $s$-wave and the gap has the same sign on all FSs [24]. In the weak coupling limit without quasiparticle renormalization in the Eliashberg theory [25], the transition temperature became unrealistically high and moreover the superconducting instability was restricted along the orbital nematic phase. These features were in sharp contrast to the typical phase diagram of FeSCs [2]. Such drawbacks were overcome by taking quasiparticle renormalizations into account [26]. The resulting onset temperature was decreased substantially down to a temperature comparable to experiments, suggesting that orbital nematic fluctuations can be a new mechanism driving high- $T_{c}$ SC. Furthermore, orbital nematic fluctuations were found to drive strong coupling SC [26]. The pairing gap was, however, assumed to be constant on each FS and thus the structure of the gap, which is the fundamental property of SC, has not been clarified.

In this paper we study the momentum dependence of the pairing gap due to orbital nematic fluctuations by employing a minimal two-band model. We solve the Eliashberg equations down to low temperatures with keeping the renormalization function. We find that the momentum dependence of SC is very weak in the tetragonal phase, whereas it becomes substantial on the hole FSs when SC occurs inside the nematic phase. These momentum dependencies are understood in terms of multiorbital natures of SC. We also find that

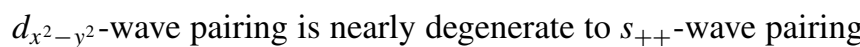
when SC occurs from the tetragonal phase, whereas such quasidegeneracy is lifted when SC occurs inside the nematic phase.

In Sec. II we describe the model and formalism. Major results are presented in Sec. III and discussed in Sec. IV. 
Conclusions are given in Sec. V. In the Appendixes we present results deeply inside the nematic phase and the gap structure associated with subleading pairing instabilities.

\section{MODEL AND FORMALISM}

To elucidate the typical feature of SC driven by orbital nematic fluctuations and to make computations feasible down to low temperatures, we employ a minimal model of orbital nematic physics. Since orbital nematic instability is described by the occupation difference between the $d_{x z}$ and $d_{y z}$ orbitals, we consider the following minimal interaction [26]:

$$
H_{I}=\frac{g}{2} \sum_{i} n_{i-} n_{i-} .
$$

Here $n_{i-}$ is the density-difference operator and is defined by $n_{i-}=n_{i 1}-n_{i 2}$ with the electron density operator $n_{i \alpha}=$ $\sum_{\sigma} c_{i \alpha \sigma}^{\dagger} c_{i \alpha \sigma} . i$ and $\sigma$ are site and spin indices, respectively, and $\alpha=1,2$ correspond to the $d_{x z}$ and $d_{y z}$ orbital, respectively. When the system retains the tetragonal symmetry, the expectation value of $n_{i-}$ becomes zero, namely $\left\langle n_{i_{-}}\right\rangle=0$. This expectation value becomes finite when the system loses $x y$ symmetry. Hence the quadratic form of $n_{i-}$ in Eq. (1) may be viewed as a typical interaction driving orbital nematicity. The coupling strength $g$ is an effective low-energy interaction coming from not only the bare intraorbital Coulomb interactions [27], but also the electron-phonon interaction [25], the Aslamazov-Larkin contribution [8], and the interorbital Coulomb interaction between $\mathrm{Fe}$ and Pnictogen [28]. In principle, the interaction (1) can lead to a nonuniform solution of $\left\langle n_{i-}\right\rangle$. However, we checked that the uniform solution gives the minimum energy in the random phase approximation for the parameters that we shall use in this paper.

The kinetic term of the two-band model may mimic the typical FSs in FeSCs [29,30]:

$$
H_{0}=\sum_{\mathbf{k}, \sigma, \alpha, \beta} \epsilon_{\mathbf{k}}^{\alpha \beta} c_{\mathbf{k} \alpha \sigma}^{\dagger} c_{\mathbf{k} \beta \sigma},
$$

where $\epsilon_{\mathbf{k}}^{11}=-2 t_{1} \cos k_{x}-2 t_{2} \cos k_{y}-4 t_{3} \cos k_{x} \cos k_{y}-\mu$, $\epsilon_{\mathbf{k}}^{22}=-2 t_{2} \cos k_{x}-2 t_{1} \cos k_{y}-4 t_{3} \cos k_{x} \cos k_{y}-\mu, \quad$ and $\epsilon_{\mathbf{k}}^{12}=-4 t_{4} \sin k_{x} \sin k_{y}$. By choosing the parameters appropriate for FeSCs [30] such as $t=-t_{1}, t_{2} / t=1.5$, $t_{3} / t=-1.2, t_{4} / t=-0.95$, and $\mu=0.6 t$, we obtain two hole FSs around $\mathbf{k}=(0,0)$ and $(\pi, \pi)$ and two electron FSs around $\mathbf{k}=(\pi, 0)$ and $(0, \pi)$ as shown in Fig. 1(a). We denote them as FS $i$ with $i=1, \ldots, 4$. FS1 and FS2 are derived from both $d_{x z}$ and $d_{y z}$ orbitals and FS3 and FS4 are from the $d_{y z}$ and the $d_{x z}$ orbital, respectively. Our FSs capture the actual orbital components obtained in the five-band model [31]. Although the $d_{x y}$ orbital is partially involved in the electron FSs, the $d_{x y}$ orbital is not relevant to orbital nematicity and thus is neglected in the present model. For simplicity we use the unit cell containing one iron.

We study the SC due to orbital nematic fluctuations in the framework of Eliashberg theory [32]. We solve the Eliashberg equations down to low temperatures with keeping the renormalization function as in the previous work [26]. The key technical development of the present work is to include a full momentum dependence of the superconducting gap on
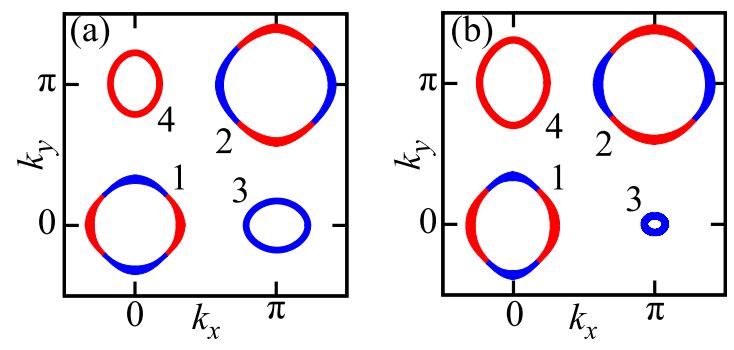

FIG. 1. Typical Fermi surfaces in the tetragonal phase (a) and the nematic phase (b). Fermi surfaces around $(0,0)$ and $(\pi, \pi)$ correspond to hole pockets and those around $(\pi, 0)$ and $(0, \pi)$ electron pockets. Each FS is denoted as FS1, FS2, FS3, and FS4, respectively. Red and blue curves denote the parts where the $d_{x z}$ and $d_{y z}$ orbital components are dominant, respectively, and the linewidth depicts its weight schematically.

each FS, which was neglected and replaced by a constant on each FS in the previous study [26].

Our pairing interaction is given by

$$
g\left(\mathbf{q}, i q_{m}\right)=g \frac{\Pi_{0}\left(\mathbf{q}, i q_{m}\right)}{1-g \Pi_{0}\left(\mathbf{q}, i q_{m}\right)} g+g_{0},
$$

where $\mathbf{q}$ and $i q_{m}$ are a momentum transfer and a bosonic Matsubara frequency, respectively. The first term is the retarded interaction mediated by nematic fluctuations and the second one accounts for instantaneous interactions from Coulomb repulsion $g_{0}>0 . \Pi_{0}\left(\mathbf{q}, i q_{m}\right)$ describes noninteracting nematic particle-hole excitations, namely $\Pi_{0}\left(\mathbf{q}, i q_{m}\right)=$ $\frac{T}{N} \sum_{\mathbf{k}, \sigma, n} \operatorname{Tr}\left[\mathcal{G}_{0}\left(\mathbf{k}, i \omega_{n}\right) \tau_{3} \mathcal{G}_{0}\left(\mathbf{k}+\mathbf{q}, i \omega_{n}+i q_{m}\right) \tau_{3}\right]$. Here $\mathcal{G}_{0}$ is a $2 \times 2$ matrix of the noninteracting Green function defined by Eq. (2), $\tau_{3}=\left(\begin{array}{cc}1 & 0 \\ 0 & -1\end{array}\right)$ is the vertex associated with the orbital nematic interaction [Eq. (1)], and $i \omega_{n}$ is a fermionic Matsubara frequency; $T$ is temperature and $N$ is the total number of lattice sites.

Since superconducting instability is a phenomenon close to the FS, we project the momenta on the FSs. We divide each FS into small patches and assign the Fermi momentum $\mathbf{k}_{F}$ on each patch; $\mathbf{k}_{F}$ is thus a discrete quantity in this paper. The resulting Eliashberg equations for the gap $\Delta\left(\mathbf{k}_{F}, i \omega_{n}\right)$ and the renormalization function $Z\left(\mathbf{k}_{F}, i \omega_{n}\right)$ then read as

$$
\begin{gathered}
\Delta\left(\mathbf{k}_{F}, i \omega_{n}\right) Z\left(\mathbf{k}_{F}, i \omega_{n}\right) \\
=-\pi T \sum_{\mathbf{k}_{F}^{\prime}, n^{\prime}} N_{\mathbf{k}_{F}^{\prime}} \frac{\tilde{g}_{\mathbf{k}_{F} \mathbf{k}_{F}^{\prime}}\left(i \omega_{n}-i \omega_{n^{\prime}}\right)}{\left|\omega_{n^{\prime}}\right|} \Delta\left(\mathbf{k}_{F}^{\prime}, i \omega_{n^{\prime}}\right), \\
Z\left(\mathbf{k}_{F}, i \omega_{n}\right)=1-\pi T \sum_{\mathbf{k}_{F}^{\prime}, n^{\prime}} N_{\mathbf{k}_{F}^{\prime}} \frac{\omega_{n^{\prime}}}{\omega_{n}} \frac{\tilde{g}_{\mathbf{k}_{F} \mathbf{k}_{F}^{\prime}}\left(i \omega_{n}-i \omega_{n^{\prime}}\right)}{\left|\omega_{n^{\prime}}\right|} .
\end{gathered}
$$

Here $\tilde{g}$ denotes effective nematic fluctuations, which are obtained by averaging the nematic fluctuations over FS patches $\mathbf{k}_{F}$ and $\mathbf{k}_{F}^{\prime}$. It is expressed by

$$
\begin{aligned}
& \tilde{g}_{\mathbf{k}_{F} \mathbf{k}_{F}^{\prime}}\left(i \omega_{n}-i \omega_{n^{\prime}}\right) \\
& =\frac{\frac{1}{N} \sum_{\mathbf{k}}^{\mathrm{FSp}} \frac{1}{N} \sum_{\mathbf{k}^{\prime}}^{\mathrm{FSp}} V\left(\mathbf{k}, \mathbf{k}^{\prime}\right)^{2} g\left(\mathbf{k}-\mathbf{k}^{\prime}, i \omega_{n}-i \omega_{n^{\prime}}\right)}{\frac{1}{N} \sum_{\mathbf{k}}^{\mathrm{FSp}} \frac{1}{N} \sum_{\mathbf{k}^{\prime}}^{\mathrm{FSp}}} .
\end{aligned}
$$

The sum over $\mathbf{k}$ is limited to a FS patch specified by $\mathbf{k}_{F}$. The vertex $V\left(\mathbf{k}, \mathbf{k}^{\prime}\right)$ describes a coupling between the 
nematic fluctuations and electrons, and is given by $V\left(\mathbf{k}, \mathbf{k}^{\prime}\right)=$ $U^{\dagger}(\mathbf{k}) \tau_{3} U\left(\mathbf{k}^{\prime}\right) ; U$ is a $2 \times 2$ unitary matrix diagonalizing the kinetic term Eq. (2). $N_{\mathbf{k}_{F}}$ in Eqs. (4) and (5) is a momentum resolved density of states defined on each FS patch. The renormalization function $Z\left(\mathbf{k}_{F}, i \omega_{n}\right)$ is frequently neglected in research of FeSCs. However, its inclusion is definitely necessary because orbital nematic fluctuations lead to a strong coupling SC [26]. As is well known, Eq. (4) can be viewed as an eigenvalue equation and the transition temperature $T_{c}$ is obtained when its eigenvalue $\lambda$ becomes unity.

\section{RESULTS}

To elucidate the typical property of SC mediated by orbital nematic fluctuations, we put $g_{0}=0$ in Eq. (3) in what follows. The major role of $g_{0}$ is to suppress $T_{c}$ as shown in Fig. 4 in Ref. [26]. In fact, the gap structure does not depend much on $g_{0}$ at least in the leading instability; appreciable $g_{0}$ dependences may appear in some subleading instabilities especially in the nematic phase. In the present model, nematic instability does not occur in $-g \lesssim 1.75 t$ [33]; the full phase diagram in the plane of temperature and the coupling constant $g$ is given in Fig. 1 in Ref. [26]. We study two typical cases: possible superconducting instability from the normal phase and from the nematic phase by taking $-g=1.7 t$ and $1.8 t$, respectively. $T_{c}$ in both cases is found to be almost the same and thus the impact of nematic order on SC can also be discussed through a comparison with the two cases.

Figure 2(a) shows the temperature dependence of the eigenvalue $\lambda$ for the five largest eigenvalues for $g=-1.7 t$ where nematic instability does not occur down to zero temperature. With decreasing temperature, all $\lambda$ increase monotonically and the largest one eventually crosses unity at $T_{c}=0.034 t$, where superconducting instability occurs.

The corresponding eigenvector at the lowest Matsubara frequency, which we denote as $\Delta_{\mathbf{k}_{F}}=\Delta\left(\mathbf{k}_{F}, i \pi T_{c}\right)$, shows $s_{++}$-wave symmetry as shown in Fig. 3(a). The gap on FS1 and FS2 exhibits a fourfold modulation, whereas the gap on FS3 and FS4 a twofold modulation even in the tetragonal phase. The modulation of the gap is very weak and is at most about $4 \%$ on the hole FS (FS2). Comparison with the orbital components of the FSs in Fig. 1(a) indicates that $\Delta_{\mathbf{k}_{F}}$ on FS1 and FS2 is slightly suppressed on the FS parts where two orbital components contribute equally. A weak modulation of the pairing gap on the hole FSs is also obtained in the spin fluctuation mechanism [34-36], which however predicts
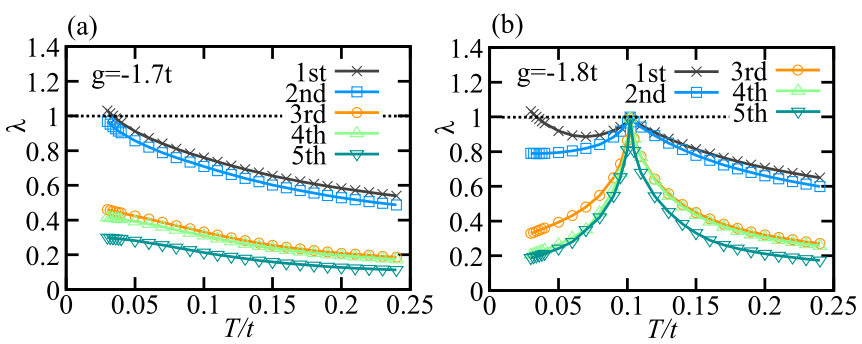

FIG. 2. Temperature dependence of the five largest eigenvalues $\lambda$ in the tetragonal phase (a) and the nematic phase (b). (a)
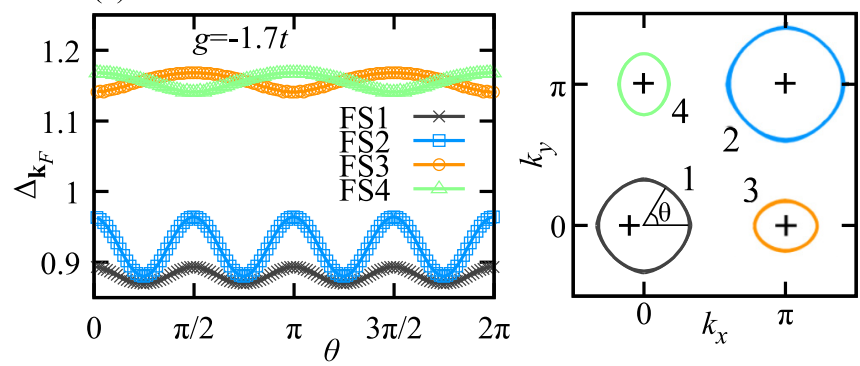

(b)
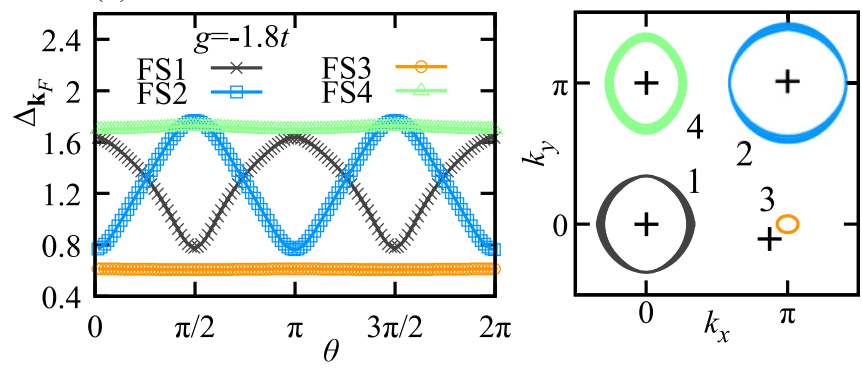

FIG. 3. Momentum dependence of the pairing gap on each FS in the tetragonal phase (a) and the nematic phase (b). In the right-hand panels, their momentum dependencies are shown schematically by featuring a gap magnitude with the thickness of each FS. The polar angle $\theta$ is measured with respect to the $k_{x}$ axis for each FS.

a large modulation of the gap on the electron FSs, in contrast to the present orbital nematic mechanism.

The second largest eigenvalue is nearly degenerate to the leading $s_{++}$-wave gap in Fig. 2(a). It corresponds to $d_{x^{2}-y^{2-}}$ wave symmetry where there are line nodes on FS1 and FS2 and a full gap on FS3 and FS4 with a sign opposite to each other. Interestingly a similar feature of such quasidegeneracy of $s$ and $d$-wave solutions was obtained also in the spin fluctuation mechanism [5,31]. In addition, the exact degeneracy between $s$ - and $d$-wave symmetry was found when nematic fluctuations are assumed independent from momentum and frequency [37]. The third, fourth, and fifth largest eigenvalues in Fig. 2(a) are rather suppressed. Details of their gap structure are presented in Fig. 6 in Appendix B.

Figure 2(b) shows the temperature dependence of eigenvalues for $g=-1.8 t$. The eigenvalues increase with decreasing $T$ and reach close to $\lambda=1$ at the onset temperature of nematic instability $T_{\mathrm{ON}}=0.102 t$. However, they do not cross unity. This peculiar behavior at $T=T_{\mathrm{ON}}$ comes from the divergence of the effective interaction $\tilde{g}_{\mathbf{k}_{F} \mathbf{k}_{F}^{\prime}}\left(i \omega_{n}-i \omega_{n^{\prime}}\right)$ at $\mathbf{k}_{F}=\mathbf{k}_{F}^{\prime}$ and $\omega_{n}=\omega_{n^{\prime}}$. As a result, $Z$ also diverges in Eq. (5). Such divergence of $\tilde{g}$ and $Z$, however, cancels out in Eq. (4) because their contributions enter like $\tilde{g} / Z$. Other nondiagonal components such as $\mathbf{k}_{F} \neq \mathbf{k}_{F}^{\prime}$ and $\omega_{n} \neq \omega_{n^{\prime}}$ in Eq. (4) become negligible compared to the diagonal components because of the contribution of $1 / Z$. Hence the matrix in Eq. (4) is reduced essentially to the unit matrix. This is the reason why all eigenvectors tend to be degenerate at the nematic transition and their eigenvalues become close to unity. In $T<T_{\mathrm{ON}}$, the nematic order develops and thus low-energy nematic fluctuations are necessarily suppressed. Consequently the eigenvalues are also suppressed. However, the largest eigenvalue starts 

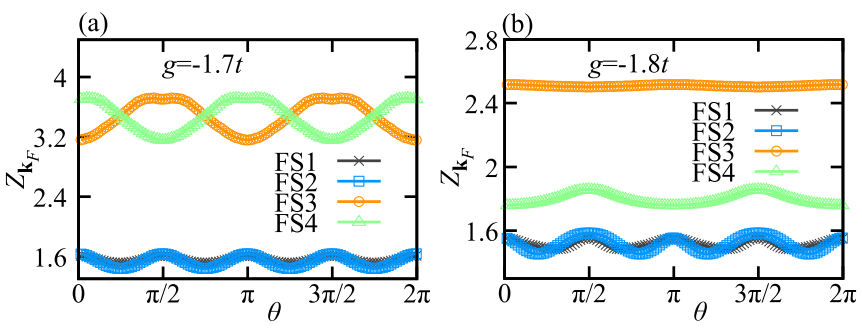

FIG. 4. Momentum dependence of the renormalization function $Z_{\mathbf{k}_{F}}$ on each FS in the tetragonal phase (a) and the nematic phase (b).

to grow again at lower temperatures, suggesting that orbital nematic fluctuations are still strong enough to drive SC. The largest eigenvalue eventually crosses unity at $T_{c}=0.034 t$, leading to superconducting instability there. In contrast to the case of superconducting instability from the tetragonal phase [Fig. 2(a)], the second largest eigenvalue, which is characterized by nodal $s$-wave symmetry [see Fig. 7(a) in Appendix B], is suppressed and no quasidegeneracy of superconducting instability occurs in the nematic phase.

While the orbital nematic order has two degenerate solutions, namely $\pm\left\langle n_{i-}\right\rangle$, we consider a positive solution here. As a result, as shown in Fig. 1(b), FS1 (FS2) elongates along the $k_{y}\left(k_{x}\right)$ direction, whereas FS4 expands along the $k_{y}$ direction and FS3 shrinks upon developing the nematic order. The corresponding eigenvector is plotted in Fig. 3(b). In contrast to Fig. 3(a), $\Delta_{\mathbf{k}_{F}}$ shows a twofold modulation on FS1 and FS2. Its modulation amounts to as large as about $40 \%$ with respect to its mean value. This strong modulation is understood in terms of the occupation difference of two orbitals in the nematic phase. In the present nematic phase, the $d_{x z}$ orbital is occupied more than the $d_{y z}$ orbital. Hence the contribution of the $d_{y z}$ orbital to the FSs becomes smaller than the other as seen in Fig. 1(b). Its contribution to the pairing is then suppressed. Since FS1 and FS2 consist of both $d_{x z}$ and $d_{y z}$ orbitals [Fig. 1(b)], the pairing gap acquires substantial modulations on the hole FSs with minima where the $d_{y z}$ orbital is dominant as shown in Fig. 3(b). It is interesting that the enhancement of the gap modulation in the nematic phase is also obtained in the spin fluctuation mechanism [38]. On the other hand, FS3 and FS4 consist of essentially a single orbital component and thus the modulation of $\Delta_{\mathbf{k}_{F}}$ remains very weak. The magnitude of the gap on FS3 becomes substantially smaller than that on FS4, because the minor $d_{y z}$ orbital forms FS3.

The momentum dependence of the renormalization function at the lowest Matsubara frequency is shown in Fig. 4; here $Z_{\mathbf{k}_{F}}=Z\left(\mathbf{k}_{F}, i \pi T_{c}\right)$. A value of $Z_{\mathbf{k}_{F}}$ is substantially larger than the typical weak-coupling SC characterized by $Z_{\mathbf{k}_{F}}$ close to unity. Hence orbital nematic fluctuations drive strong coupling SC [26]. On FS1 and FS2, $Z_{\mathbf{k}_{F}}$ shows a weak $\mathbf{k}_{F}$ dependence in both tetragonal and nematic phases, with fourfold symmetry in the former and twofold symmetry in the later. On FS3 and FS4, $Z_{\mathbf{k}_{F}}$ exhibits a twofold modulation and its value is enhanced more than that on FS1 and FS2. This is because the size of the FS is rather small and thus orbital nematic fluctuations, which have large spectral weight at small momentum, contribute effectively via intrapocket scattering processes. In particular, the value of $Z_{\mathbf{k}_{F}}$ amounts to as large as about 3.7 in the tetragonal phase.
While we have considered the case where FS3 survives in the nematic phase, essentially the same results are obtained even if FS3 disappears due to large nematicity. Details are presented in Appendix A.

\section{DISCUSSIONS}

We have studied SC mediated by orbital nematic fluctuations. In particular, we have elucidated one of fundamental properties of SC, namely the structure of the pairing gap, by allowing a momentum dependence of the gap in a theory of Ref. [26]. Except that the pairing gap and the renormalization function acquire a momentum dependence, we have found that results obtained in Ref. [26] remain valid almost quantitatively. In fact, a value of $T_{c}$ is enhanced only slightly by allowing the momentum dependence.

The structure of the pairing gap can be revealed directly by angle-resolved photoemission spectroscopy (ARPES). We have obtained the weak momentum dependence of the gap in the tetragonal phase [Fig. 3(a)], which can be viewed as a nearly isotropic gap. Such a gap roughly captures experimental observations in various materials when we focus on the FSs originating mainly from the $d_{x z}$ and $d_{y z}$ orbitals: $\mathrm{BaFe}_{2}\left(\mathrm{As}_{1-x} \mathrm{P}_{x}\right)_{2}$ with $x=0.30$ [39] and 0.35 [40], $\mathrm{Ba}_{0.6} \mathrm{~K}_{0.4} \mathrm{Fe}_{2} \mathrm{As}_{2}$ [40], and $\mathrm{FeTe}_{0.6} \mathrm{Se}_{0.4}$ [41]. While we have obtained $s_{++}$-wave symmetry as the leading instability, the sign of the superconducting gap can be a subtle issue. In the mechanism of orbital nematic fluctuations, $\mathrm{SC}$ is driven mainly by intrapocket scattering processes and the relative sign among the gaps on different pockets is determined by interpocket nematic fluctuations which are relatively weak [26]. Hence the pairing symmetry could easily change from $s_{++}$to $s_{ \pm}$wave when some repulsive interactions between hole and electron pockets are added even if orbital nematic fluctuations are still dominant. This is actually the case when spin fluctuations are added to orbital nematic fluctuations [42] and to various charge fluctuations [28].

For $\mathrm{Ba}\left(\mathrm{Fe}_{1-x} \mathrm{Co}_{x}\right)_{2} \mathrm{As}_{2}$ with $x=0.1$, a nearly isotropic gap was observed on all FSs except for one electron FS around $(\pi, 0)$, where nodes or gap minima were reported [43]. The presence of the nodelike structure in the tetragonal phase is not captured in the present theory, which may be resolved by considering the following possibilities. First, while the $d_{x z}$ and $d_{y z}$ orbitals are dominant contributions to the Fermi level, the second dominant contribution comes from the $d_{x y}$ orbital [31]. Since a modulation of the gap originates from the multiorbital natures in the present theory, a stronger modulation, namely gap minima, could be realized by including the $d_{x y}$ orbital. Second, the leading $s_{++}$-wave symmetry is nearly degenerate to the second leading instability [Fig. 3(a)], which is characterized by $d_{x^{2}-y^{2}}$-wave symmetry. This $d_{x^{2}-y^{2}}$-wave symmetry could be stabilized by additional effects such as spin fluctuations. Third, spin fluctuations themselves, on the other hand, tend to drive $s_{ \pm}$-wave symmetry in general [4-6] and their inclusion yields the competition with $s_{++}$-wave symmetry. Such competition may lead to a nodelike feature of $s$-wave gap. This is indeed the case at least when the system contains both orbital fluctuations with large momentum transfers and spin fluctuations [44]. While a node was not obtained, the pairing gap was found to exhibit 
large modulations on the electron FSs when spin and charge fluctuations compete with each other; the gap on the hole FSs, however, remains almost isotropic [28].

The superconducting gap structure was also revealed for FeSe films, whose $T_{c}$ can be more than $65 \mathrm{~K}$ [45-47]. A nearly isotropic gap was observed on the electron FSs for monolayer FeSe [48,49] and K-coated multilayer FeSe [50]. A similar gap structure was also observed for $\mathrm{Cs}_{0.8} \mathrm{Fe}_{2} \mathrm{Se}_{2}$ [51] and $\mathrm{K}_{0.8} \mathrm{Fe}_{2} \mathrm{Se}_{2}$ [51]. These results are consistent with our results [Fig. 3(a)]. While FeSe films [46] and alkali-intercalated FeSe [51] are special in the sense that hole FSs are absent and only the electron FSs exist, the present theory is expected not to be sensitive to the actual FS geometry (see also Appendix A) since SC from orbital nematic fluctuations comes mainly from intrapocket scattering processes [26].

A superconducting gap inside the nematic phase may be discussed for $\mathrm{FeSe}_{0.93} \mathrm{~S}_{0.07}$ [52]. The gap on the hole FS around $(0,0)$ exhibits the sizable momentum dependence with gap maxima (minima) along the $k_{x}\left(k_{y}\right)$ axis in Fig. 3(b), which captures the observed gap structure on the hole FS [52]. The SC gap on the hole FS around $(\pi, \pi)$ has maxima (minima) along the $k_{y}\left(k_{x}\right)$ direction as seen in Fig. 3(b). Recalling that FeSCs have two irons per unit cell, our Brillouin zone would be folded and thus the hole FS around $(\pi, \pi)$ is actually moved around $(0,0)$ through a momentum shift of $(\pi, \pi)$, forming the outer hole FS around $(0,0)$. Consequently, we expect an antiphase gap structure between two hole FSs around $(0,0)$, that is, the outer FS has a larger (smaller) gap along the $k_{y}\left(k_{x}\right)$ axis, whereas the inner FS has a smaller (larger) gap there. This predicted gap structure as well as the gap on the electron FSs has not been resolved in experiments [52].

\section{CONCLUSIONS}

Employing a minimal two-band model consisting of the $d_{x z}$ and $d_{y z}$ orbitals, we have studied typical properties of SC mediated by orbital nematic fluctuations. We have solved the Eliashberg equations down to the superconducting onset temperature with keeping not only the renormalization function but also a full momentum dependence of the paring gap on the FSs. We have found that the leading instability is $s_{++}$-wave symmetry. The pairing gap exhibits a fourfold and twofold modulation on the hole and electron FSs, respectively, in the tetragonal phase. The gap is suppressed on the parts of the FSs where two orbitals contribute equally, but its suppression is weak and the gap may be approximated as a constant. SC with $d_{x^{2}-y^{2}}$-wave symmetry can also be driven by orbital nematic fluctuations as a nearly degenerate state to the $s_{++}$-wave state. The impact of the nematic order is noticeable. First, the gap on the hole FSs acquires a significant modulation. The gap is suppressed on parts of the FSs where the $d_{y z}\left(d_{x z}\right)$ orbital becomes dominant, when the $d_{y z}\left(d_{x z}\right)$ orbital is occupied less than the other. Second, the fourfold modulation of the gap on the hole FSs changes to a twofold modulation, whereas the twofold modulation remains on the electron FSs. Third, the quasidegeneracy of $s_{++}$and $d_{x^{2}-y^{2}}$-wave solutions is lifted in the nematic phase. The $d_{x^{2}-y^{2}}$-wave solution is suppressed by changing its symmetry to highly anisotropic $s$-wave state.

We have focused on orbital nematic fluctuations in order to establish the typical gap structure of SC mediated by them, which will serve to disentangle complex phenomena with combined effects from multiorbitals and multifluctuations in FeSCs. Given that the nematic phase is realized close to the SDW phase in the general phase diagram of FeSCs, we consider it reasonable to assume that spin fluctuations are also important to SC. In fact, there are a plenty of studies trying to explain the superconducting gap in FeSCs in terms of the spin fluctuation mechanism [53]. An important future issue is to clarify the condition of which mechanism, spin fluctuations or orbital nematic fluctuations, is dominant over the other or whether both mechanisms should be considered on an equal footing in general. Although these two mechanisms rely on different physics, interestingly they share some aspects of SC: (i) the pairing gap with $s$-wave symmetry [4-6], (ii) the presence of a $d_{x^{2}-y^{2}}$-wave solution nearly degenerate to the leading instability in the tetragonal phase [5,31], (iii) the weak modulation of the pairing gap on the hole FSs in the tetragonal phase [34-36], and (iv) its enhancement in the nematic phase [38]. The positions of gap minima/maxima obtained in the spin fluctuation mechanism [38], however, vary from the present work.

\section{ACKNOWLEDGMENTS}

The authors thank T. Shimojima for valuable discussions on unpublished data in his group and T. Terashima for many fruitful comments. This work was supported by JSPS KAKENHI Grant No. 15K05189.

\section{APPENDIX A: GAP STRUCTURE DEEPLY INSIDE THE NEMATIC PHASE}

Superconductivity mediated by orbital nematic fluctuations comes mainly from intrapocket scattering processes [26]. Hence the geometry of the FSs is not important to the superconducting instability. This is a crucial difference from other superconducting mechanisms such as spin fluctuations [4-6] and orbital fluctuations with a large momentum transfer $[7,8]$. To demonstrate this, we here present results of superconducting instability deeply inside the nematic phase $(g=-1.9 t)$, where FS3 vanishes due to large nematicity and the other FSs, namely FS1, FS2, and FS4 are elongated slightly more than Fig. 1(b), as seen in Fig. 5(d).

Figure 5(a) shows the temperature dependence of the eigenvalues. With decreasing temperature, the eigenvalues increase and take a cusp at $T=T_{\mathrm{ON}}=0.202 t$, where the nematic instability sets in. The eigenvalues do not cross unity there because the quasiparticle residue $Z^{-1}$ goes to zero there, as in the case of Fig. 2(b). Below $T_{\mathrm{ON}}$, low-energy orbital nematic fluctuations are suppressed, leading to the suppression of the eigenvalues. At $T \approx 0.14 t$, the eigenvalues drop discontinuously. This temperature corresponds to the temperature at which FS3 vanishes because the nematic order parameter grows to be large enough to push up FS3 above the Fermi energy. The largest eigenvalue, however, starts to increase at lower temperature and finally leads to superconducting instability at $T_{c}=0.052 t$.

In Fig. 5(c) we show the momentum dependence of the pairing gap. The results are essentially the same as Fig. 3(b). A quantitative difference is that $\Delta_{\mathbf{k}_{F}}$ acquires a larger modulation 

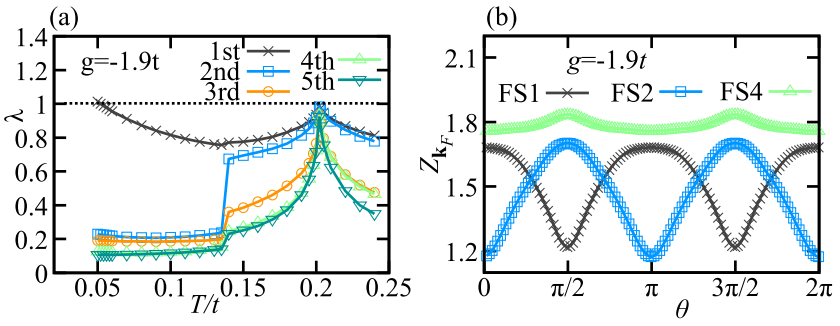

(c)

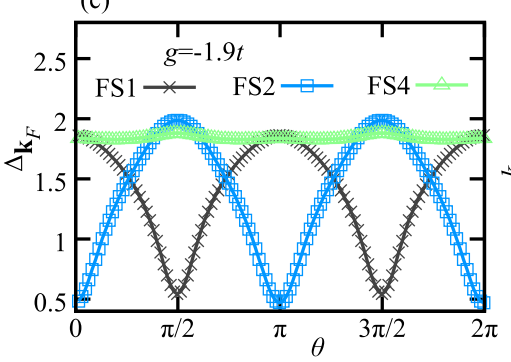

(d)

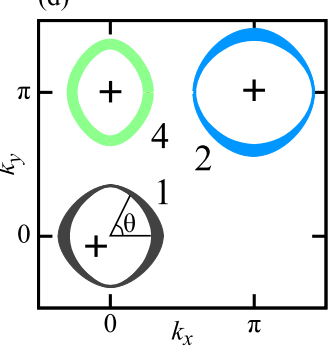

FIG. 5. Major results deeply inside the nematic phase for $g=$ $-1.9 t$ where FS3 vanishes. (a) Temperature dependence of the five largest eigenvalues $\lambda$. (b) Momentum dependence of the renormalization function $Z_{\mathbf{k}_{F}}$ on each FS. The polar angle $\theta$ is measured with respect to the $k_{x}$ axis for each FS as shown in (d). (c) Momentum dependence of the pairing gap on each FS. (d) Sketch of $\Delta_{\mathbf{k}_{F}}$ by featuring a gap magnitude with the thickness of each FS.

on the hole FSs. The regions on the FSs where the $d_{x z}$ and $d_{y z}$ orbital components are dominant are almost the same as Fig. 1(b) except for the absence of FS3. The gap minima are then realized on the FS parts consisting mainly of the minority orbital, namely the $d_{y z}$ component. The resulting modulation of the pairing gap amounts to as large as about $60 \%$. In spite of the large modulations on FS1 and FS2, the

(a) Second largest
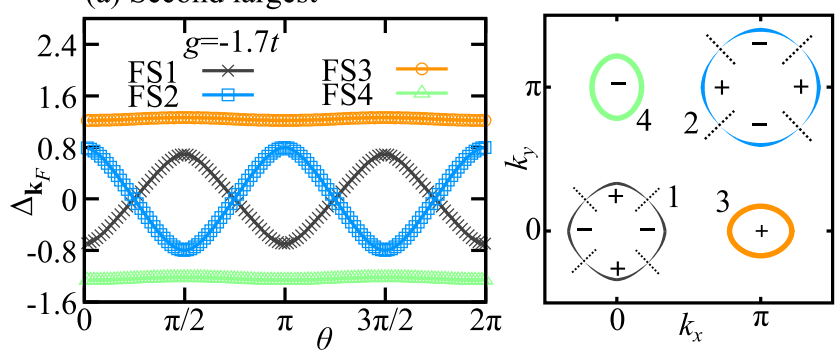

(c) Fourth largest
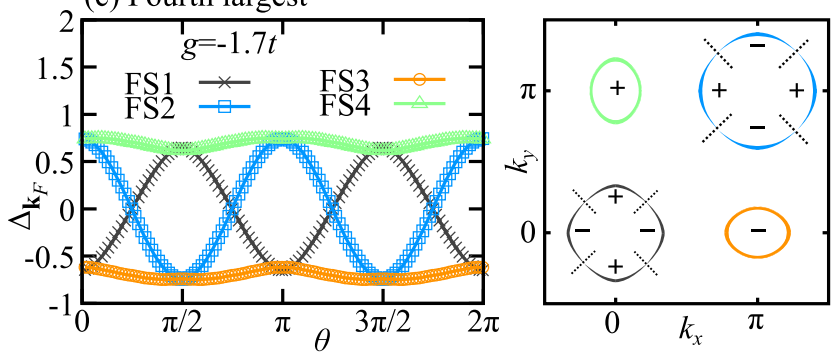

gap on FS4 exhibits a very weak momentum dependence. This is because FS4 consists of essentially a single orbital (see Fig. 1). We summarize the gap structure associated with subleading instabilities in Fig. 8 in Appendix B.

The corresponding renormalization function is shown in Fig. 5(b). In line with the large modulation of $\Delta_{\mathbf{k}_{F}}$ on FS1 and FS2, $Z_{\mathbf{k}_{F}}$ on FS1 and FS2 also shows a modulation as large as about $17 \%$ with a twofold modulation much more clearly than the corresponding results in Fig. 4(b) because of larger nematicity here. On the other hand, $Z_{\mathbf{k}_{F}}$ on FS4 features a very weak momentum dependence, similar to that of $\Delta_{\mathbf{k}_{F}}$.

\section{APPENDIX B: MOMENTUM DEPENDENCE OF PAIRING GAP OF SUBLEADING INSTABILITIES}

We present the momentum dependence of the pairing gap associated with the second, third, fourth, and fifth largest eigenvalues shown in Figs. 2 and 5(a).

Figure 6 shows results in the tetragonal phase $(g=-1.7 t)$. The second largest eigenvalue is characterized by $d_{x^{2}-y^{2}}$-wave symmetry, which is nearly degenerate to the leading $s_{++}$-wave symmetry [see Fig. 2(a)]. The third one is characterized by $s_{ \pm}$-wave symmetry, which is the same symmetry as that often obtained in the spin fluctuation mechanism [4-6]. The fourth

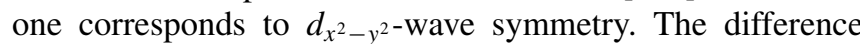
from the second one lies in the sign of the gap on FS3 and FS4. The fifth one is characterized by $s_{++}$-wave symmetry, the same symmetry as the leading one [Fig. 3(a)]. The main difference appears in the magnitude of the gap on FS3 and FS4, which is substantially suppressed for the fifth leading instability. Looking through those gap structure of the subleading instabilities, we can conclude that the momentum dependence of the pairing gap is very weak for the $s$-wave solutions in the tetragonal phase.

(b) Third largest
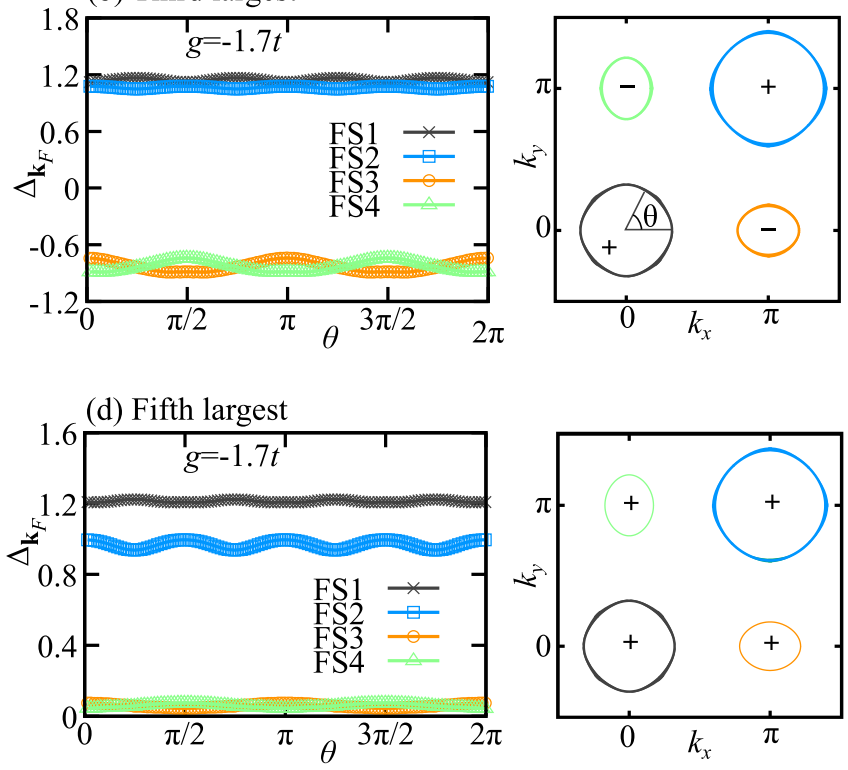

FIG. 6. Momentum dependence of the pairing gap for the second (a), third (b), fourth (c), and fifth (d) largest eigenvalues in the tetragonal phase for $g=-1.7 t$. The right-hand panel is a sketch of the gap structure by featuring a gap magnitude with the thickness of each FS. The polar angle $\theta$ is measured with respect to the $k_{x}$ axis for each FS. 
(a) Second largest

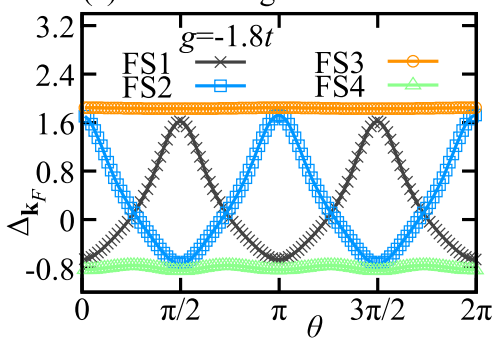

(c) Fourth largest

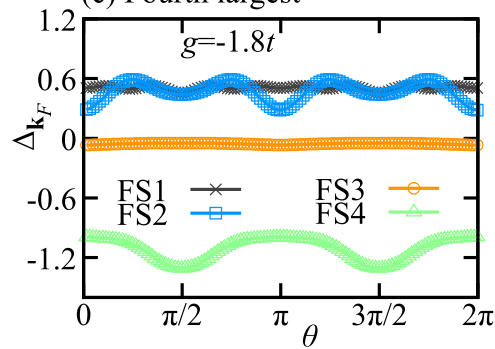

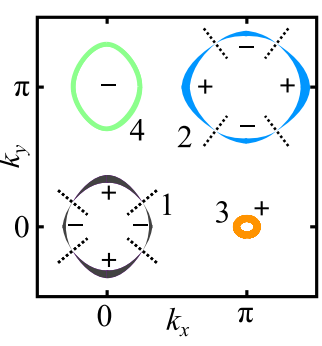

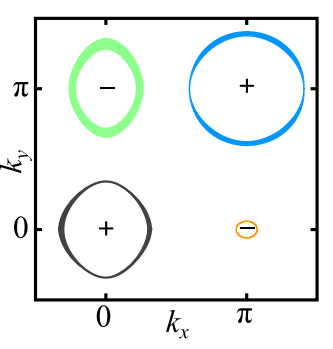

(b) Third largest
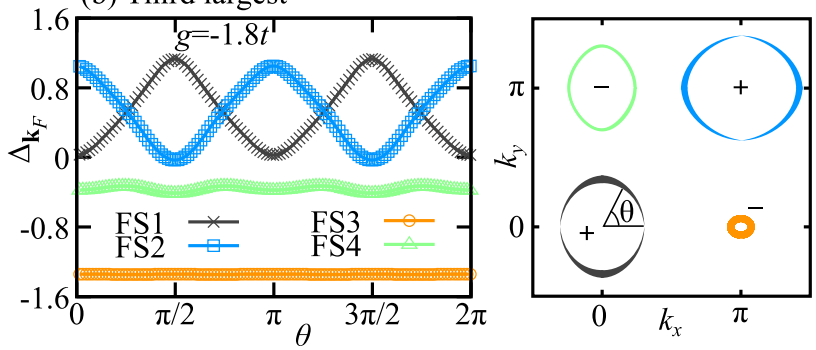

(d) Fifth largest
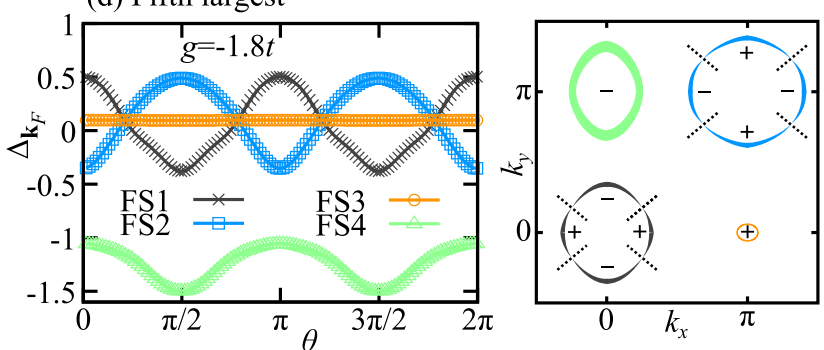

FIG. 7. Momentum dependence of the pairing gap for the second (a), third (b), fourth (c), and fifth (d) largest eigenvalues in the nematic phase for $g=-1.8 t$. The right-hand panel is a sketch of the gap structure by featuring a gap magnitude with the thickness of each FS.

In the nematic phase the pairing gap acquires a sizable modulation along the FSs. Figure 7 is the corresponding results in the nematic phase for $g=-1.8 t$. The pairing gap for the second largest eigenvalue shows a similar momentum dependence to that in Fig. 6(a). However, $d_{x^{2}-y^{2}}$-wave symmetry cannot be defined in the nematic phase. Instead, the result in Fig. 7(a) is characterized by nodal $s$-wave symmetry. Nodes enter hole pockets FS1 and FS2. The third leading instability corresponds to the so-called $s_{ \pm}$-wave symmetry although $s$-wave gap on FS1 and FS2 becomes nearly zero at $\theta=0, \pi$ and $\theta=\pi / 2,3 \pi / 2$, respectively. It is interesting that the pairing gap on FS3 becomes largest in Figs. 7(a) and 7(b), although FS3 is tiny. The fourth one is also characterized by $s_{ \pm}$-wave symmetry. In contrast to the third one, the gap on FS3 is nearly zero. While this is not a leading instability, the solution in Fig. 7(c) provides an interesting example of essentially gapless $s$-wave SC in a multipocket system. The fifth solution [Fig. 7(d)] is similar to the fourth one in the tetragonal phase [Fig. 6(c)] and features a kind of $d_{x^{2}-y^{2}}$-wave symmetry, although the correct symmetry is the so-called (a) Second largest
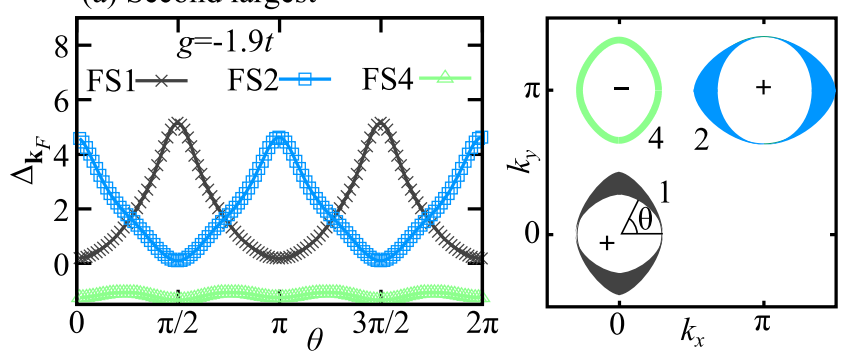

(c) Fourth largest
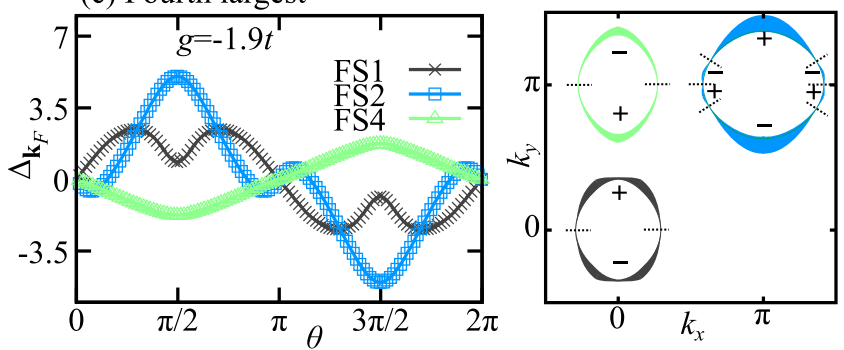

(b) Third largest
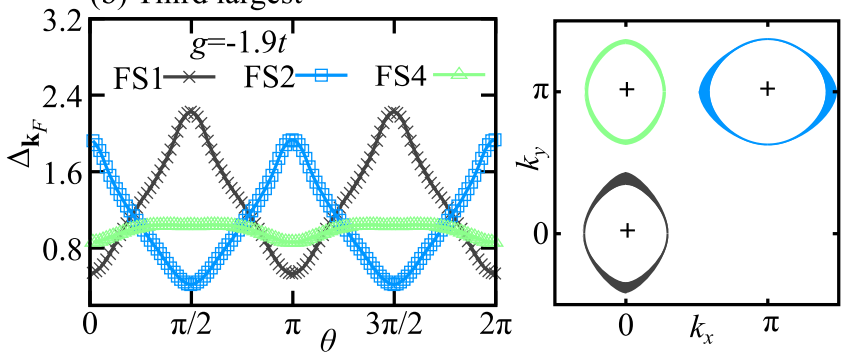

(d) Fifth largest
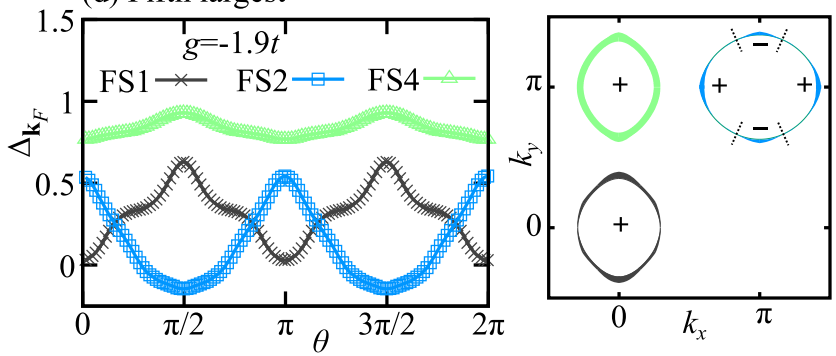

FIG. 8. Momentum dependence of the pairing gap for the second (a), third (b), fourth (c), and fifth (d) largest eigenvalues deeply inside the nematic phase for $g=-1.9 t$ where FS3 vanishes. The right-hand panel is a sketch of the gap structure by featuring a gap magnitude with the thickness of each FS. 
$s$-wave symmetry classified by $A_{1}$ representation in the $C_{2 v}$ point group. The gap on FS3 is nearly zero, similar to the case of the fourth solution [Fig. 7(c)].

While the leading instability is characterized by the same $s_{++}$-wave symmetry in both Figs. 3(b) and 5(c), the subleading instabilities deeply inside the nematic phase, where FS3 vanishes, exhibit symmetries rather different from Fig. 7. Figure 8(a) shows $\Delta_{\mathbf{k}_{F}}$ corresponding to the second largest eigenvalue. It is characterized by a very large modulation on FS1 and FS2 and the gap almost vanishes at $\theta=0, \pi$ on FS1 and $\theta=\pi / 2,3 \pi / 2$ on FS2. This solution is similar to $\Delta_{\mathbf{k}_{F}}$ of the third largest eigenvalue for $g=-1.8 t$ [see Fig. 7(b)]. The gap on FS4 has the sign opposite to that on the hole
FSs. In this sense the gap structure is $s_{ \pm}$-wave symmetry. Figure 8(b) corresponds to the third largest eigenvalue and is a similar result to Fig. 8(a), except that the gap on FS4 has the opposite sign and the modulation of the gaps on FS1 and FS2 is smaller. In fact, these two solutions are almost degenerate as seen in Fig. 5(a). The fourth largest eigenvalue corresponds to $p$-wave symmetry, as shown in Fig. 8(c). It is interesting to recognize that a $p$-wave solution, in principle, can be driven orbital nematic fluctuations deeply inside the nematic phase. This $p$-wave solution is almost degenerate to the fifth leading instability as seen in Fig. 5(a). The fifth one is nodal $s$-wave symmetry with nodes on FS2. A nodelike feature is also realized on FS1 where the gap nearly vanishes at $\theta=0, \pi$.
[1] Y. Kamihara, T. Watanabe, M. Hirano, and H. Hosono, J. Am. Chem. Soc. 130, 3296 (2008).

[2] G. R. Stewart, Rev. Mod. Phys. 83, 1589 (2011).

[3] I. R. Fisher, L. Degiorgi, and Z. X. Shen, Rep. Prog. Phys. 74, 124506 (2011).

[4] I. I. Mazin, D. J. Singh, M. D. Johannes, and M. H. Du, Phys. Rev. Lett. 101, 057003 (2008).

[5] K. Kuroki, S. Onari, R. Arita, H. Usui, Y. Tanaka, H. Kontani, and H. Aoki, Phys. Rev. Lett. 101, 087004 (2008).

[6] A. V. Chubukov, D. V. Efremov, and I. Eremin, Phys. Rev. B 78, 134512 (2008).

[7] T. D. Stanescu, V. Galitski, and S. DasSarma, Phys. Rev. B 78, 195114 (2008).

[8] H. Kontani, T. Saito, and S. Onari, Phys. Rev. B 84, 024528 (2011).

[9] Y. Gallais, R. M. Fernandes, I. Paul, L. Chauvière, Y. X. Yang, M. A. Méasson, M. Cazayous, A. Sacuto, D. Colson, and A. Forget, Phys. Rev. Lett. 111, 267001 (2013).

[10] R. M. Fernandes, A. V. Chubukov, and J. Schmalian, Nat. Phys. 10, 97 (2014).

[11] S. A. Kivelson, E. Fradkin, and V. J. Emery, Nature (London) 393, 550 (1998).

[12] H. Yamase and H. Kohno, J. Phys. Soc. Jpn. 69, 332 (2000); 69, 2151 (2000)

[13] C. J. Halboth and W. Metzner, Phys. Rev. Lett. 85, 5162 (2000).

[14] A. F. Andreev and I. A. Grishchuk, Zh. Eksp. Teor. Fiz. 87, 467 (1984) [Sov. Phys. JETP 60, 267 (1984)].

[15] S. Raghu, A. Paramekanti, E-.A. Kim, R. A. Borzi, S. A. Grigera, A. P. Mackenzie, and S. A. Kivelson, Phys. Rev. B 79, 214402 (2009)

[16] W.-C. Lee and C. Wu, Phys. Rev. B 80, 104438 (2009).

[17] C. Fang, H. Yao, W.-F. Tsai, J. P. Hu, and S. A. Kivelson, Phys. Rev. B 77, 224509 (2008).

[18] C. Xu, Y. Qi, and S. Sachdev, Phys. Rev. B 78, 134507 (2008).

[19] F. Krüger, S. Kumar, J. Zaanen, and J. van den Brink, Phys. Rev. B 79, 054504 (2009).

[20] C.-C. Lee, W.-G. Yin, and W. Ku, Phys. Rev. Lett. 103, 267001 (2009).

[21] W. Lv, J. Wu, and P. Phillips, Phys. Rev. B 80, 224506 (2009).

[22] S.-H. Baek, D. V. Efremov, J. M. Ok, J. S. Kim, J. van den Brink, and B. Büchner, Nat. Mater. 14, 210 (2015).

[23] H. Yamase and R. Zeyher, New J. Phys. 17, 073030 (2015).
[24] Y. Yanagi, Y. Yamakawa, and Y. Ōno, Phys. Rev. B 81, 054518 (2010).

[25] Y. Yanagi, Y. Yamakawa, N. Adachi, and Y. Ōno, J. Phys. Soc. Jpn. 79, 123707 (2010).

[26] H. Yamase and R. Zeyher, Phys. Rev. B 88, 180502(R) (2013).

[27] In the two-band Hubbard model the coupling strength $g$ is expressed by $g=U / 4-U^{\prime} / 2+J / 4$, where $U$ and $U^{\prime}$ are the intra- and interorbital Coulomb interaction, respectively, and $J$ is the Hund coupling.

[28] S. Zhou, G. Kotliar, and Z. Wang, Phys. Rev. B 84, 140505(R) (2011).

[29] S. Raghu, X.-L. Qi, C.-X. Liu, D. J. Scalapino, and S.-C. Zhang, Phys. Rev. B 77, 220503 (2008).

[30] Z.-J. Yao, J.-X. Li, and Z. D. Wang, New J. Phys. 11, 025009 (2009).

[31] S. Graser, T. M. Maier, P. J. Hirschfeld, and D. J. Scalapino, New J. Phys. 11, 025016 (2009).

[32] J. R. Schrieffer, Theory of Superconductivity, revised ed. (Perseus Books, New York, 1999).

[33] Our phase boundary is not exactly the same as Fig. 1 in Ref. [26]. This is because our numerical accuracy to compute Eq. (3) had to be compromised over respecting fine $\mathbf{k}_{F}$ and $\mathbf{k}_{F}^{\prime}$ dependencies of $\tilde{g}$ in Eq. (6).

[34] T. A. Maier, S. Graser, D. J. Scalapino, and P. J. Hirschfeld, Phys. Rev. B 79, 224510 (2009).

[35] R. Thomale, C. Platt, W. Hanke, and B. A. Bernevig, Phys. Rev. Lett. 106, 187003 (2011).

[36] S. Maiti, M. M. Korshunov, T. A. Maier, P. J. Hirschfeld, and A. V. Chubukov, Phys. Rev. B 84, 224505 (2011).

[37] J. Kang and R. M. Fernandes, Phys. Rev. Lett. 117, 217003 (2016).

[38] J. Kang, A. F. Kemper, and R. M. Fernandes, Phys. Rev. Lett. 113, 217001 (2014).

[39] T. Yoshida, S. Ideta, T. Shimojima, W. Malaeb, K. Shinada, H. Suzuki, I. Nishi, A. Fujimori, K. Ishizaka, S. Shin, Y. Nakashima, H. Anzai, M. Arita, A. Ino, H. Namatame, M. Taniguchi, H. Kumigashira, K. Ono, S. Kasahara, T. Shibauchi, T. Terashima, Y. Matsuda, M. Nakajima, S. Uchida, Y. Tomioka, T. Ito, K. Kihou, C. H. Lee, A. Iyo, H. Eisaki, H. Ikeda, R. Arita, T. Saito, S. Onari, and H. Kontani, Sci. Rep. 4, 7292 (2014).

[40] T. Shimojima, F. Sakaguchi, K. Ishizaka, Y. Ishida, T. Kiss, M. Okawa, T. Togashi, C.-T. Chen, S. Watanabe, M. Arita, K. 
Shimada, H. Namatame, M. Taniguchi, K. Ohgushi, S. Kasahara, T. Terashima, T. Shibauchi, Y. Matsuda, A. Chainani, and S. Shin, Science 332, 564 (2011).

[41] K. Okazaki, Y. Ito, Y. Ota, Y. Kotani, T. Shimojima, T. Kiss, S. Watanabe, C.-T. Chen, S. Niitaka, T. Hanaguri, H. Takagi, A. Chainani, and S. Shin, Phys. Rev. Lett. 109, 237011 (2012).

[42] T. Yamada, J. Ishizuka, and Y. Ōno, J. Phys. Soc. Jpn. 83, 043704 (2014).

[43] T. Hajiri, T. Ito, M. Matsunami, B. H. Min, Y. S. Kwon, and S. Kimura, J. Phys. Soc. Jpn. 83, 093703 (2014).

[44] T. Saito, S. Onari, and H. Kontani, Phys. Rev. B 88, 045115 (2013).

[45] Q.-Y. Wang, Z. Li, W.-H. Zhang, Z.-C. Zhang, J.-S. Zhang, W. Li, H. Ding, Y.-B. Ou, P. Deng, K. Chang, J. Wen, C.-L. Song, K. He, J.-F. Jia, S.-H. Ji, Y. Wang, L. Wang, X. Chen, X. Ma, and Q.-K. Xue, Chin. Phys. Lett. 29, 037402 (2012).

[46] S. He, J. He, W. Zhang, L. Zhao, D. Liu, X. Liu, D. Mou, Y.-B. Ou, Q.-Y. Wang, Z. Li, L. Wang, Y. Peng, Y. Liu, C. Chen, L. Yu, G. Liu, X. Dong, J. Zhang, C. Chen, Z. Xu, X. Chen, X. Ma, Q. Xue, and X. J. Zhou, Nat. Mater. 12, 605 (2013).
[47] J.-F. Ge, Z.-L. Liu, C. Liu, C.-L. Gao, D. Qian, Q.-K. Xue, Y. Liu, and J.-F. Jia, Nat. Mater. 14, 285 (2015).

[48] D. Liu, W. Zhang, D. Mou, J. He, Y.-B. Ou, Q.-Y. Wang, Z. Li, L. Wang, L. Zhao, S. He, Y. Peng, X. Liu, C. Chen, L. Yu, G. Liu, X. Dong, J. Zhang, C. Chen, Z. Xu, J. Hu, X. Chen, X. Ma, Q. Xue, and X. J. Zhou, Nat. Commun. 3, 931 (2012).

[49] Y. Zhang, J. J. Lee, R. G. Moore, W. Li, M. Yi, M. Hashimoto, D. H. Lu, T. P. Devereaux, D.-H. Lee, and Z.-X. Shen, Phys. Rev. Lett. 117, 117001 (2016).

[50] Y. Miyata, K. Nakayama, K. Sugawara, T. Sato, and T. Takahashi, Nat. Mater. 14, 775 (2015).

[51] Y. Zhang, L. X. Yang, M. Xu, Z. R. Ye, F. Chen, C. He, H. C. Xu, J. Jiang, B. P. Xie, J. J. Ying, X. F. Wang, X. H. Chen, J. P. $\mathrm{Hu}, \mathrm{M}$. Matsunami, S. Kimura, and D. L. Feng, Nat. Mater. 10, 273 (2011).

[52] H. C. Xu, X. H. Niu, D. F. Xu, J. Jiang, Q. Yao, Q. Y. Chen, Q. Song, M. Abdel-Hafiez, D. A. Chareev, A. N. Vasiliev, Q. S. Wang, H. L. Wo, J. Zhao, R. Peng, and D. L. Feng, Phys. Rev. Lett. 117, 157003 (2016).

[53] P. J. Hirschfeld, C. R. Phys. 17, 197 (2016). 